\title{
42 Obesity and the risk for disease
}

In today's society with its nearly unlimited nutritional resources, the survival benefit achieved through the body's ability to store large fat reserves has been reversed (van Cleave et al. 2010). Overweight and obesity are contributing causes to very serious health problems. These include, among others, senile dementia ( $\downarrow$ Chapter 38 ), cancer, hypertension, diabetes mellitus and an elevated incidence of arteriosclerotic complications such as myocardial infarction and stroke (Chen et al. 2013, $\mathrm{Lu}$ et al. 2014). As demonstrated by the data from a study on 302,296 persons in Europe and the USA with observation periods of 6-35 years, the associated risk, especially for coronary heart disease, increases by $17 \%$ in persons with a BMI between 25 and 29.9 and by $45 \%$ with a BMI $>30$. These figures have already been corrected for the risks of high blood pressure and cholesterol levels (Bogers et al. 2007).

The concurrent presence of cardiovascular diseases and diabetes approximately doubles the risk of death. This association was revealed by the interpretation of a meta-analysis (data from comparable studies are amalgamated to strengthen the conclusiveness of their findings) from 102 prospective studies with close to 700,000 patients (ERFC 2010).

The findings of the Nurses' Health Study reinforce the importance of maintaining a normal body weight, starting early in adulthood. In women, extreme obesity at the age of 50 years dramatically limits the perspective for a good state of health at an older age of 70 years (Sun et al. 2009). If obesity

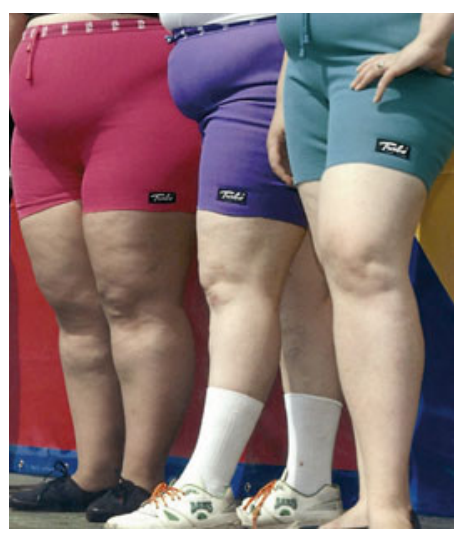

- Fig. 42.1 Source: dpa/akg

sets in at the early age of 18 , the chances of staying healthy and strong in older age decrease even further. Each kilogram of weight gain after the 18th year of life decreases these chances by $5 \%$.

One particular characteristic is repeatedly described by larger studies. In patients who are already severely ill, e.g. suffer from chronic kidney disease, diabetes, cardiovascular disease or cancer, the ones with a higher BMI will often have better survival chances than leaner patients (Carnethon et al. 2012, Sharma et al. 2014). This phenomenon is termed the obesity paradox in the literature. Many theories have been suggested to explain the underlying physiology, but it still remains unclear (Angeras et al. 2013, von Haehling et al. 2013, de Schutter et al. 2014). 